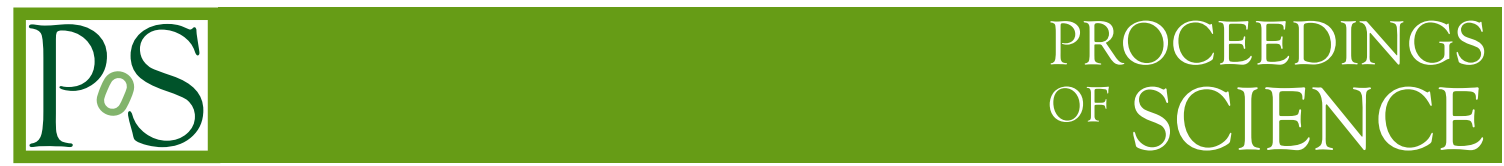

\title{
Eguchi-Kawai model with dynamical adjoint fermions
}

\section{Ari Hietanen*}

Florida International University

E-mail: ari.hietanenefiu.edu

\section{Rajamana Narayanan}

Florida International University

E-mail: rajamani.narayananefiu.edu

\begin{abstract}
It is believed that fermions in adjoint representation on single site lattice will restore the center symmetry, which is a crucial requirement for the volume independence of large-N lattice gauge theories. We present a perturbative analysis which supports the assumption for overlap fermions, but shows that center symmetry is broken for naive fermions.
\end{abstract}

The XXVII International Symposium on Lattice Field Theory - LAT2009

July 26-31 2009

Peking University, Beijing, China

${ }^{*}$ Speaker. 


\section{Introduction}

Eguchi and Kawai proposed [1] that a pure gauge $\mathrm{SU}(N)$ lattice theory can be reduced to a single site at the limit $N \rightarrow \infty$. The proof was based on the assumption that the single site has a center symmetry associated with the traces of the Polyakov loops. However, later the center symmetry was shown to be broken in the continuum limit [2].

It was immediately clear that EK-reduction might work for theories with supersymmetry [3]. This has been explored recently in the continuum [4], where it was suggested that fermions obeying periodic boundary conditions in the adjoint representation, QCD(Adj), would be volume independent. A continuum perturbation theory analysis on $\mathbf{R}^{3} \times S^{1}$ showed $Z_{N}$ symmetry to be restored. Similar calculations on $S^{3} \times S^{1}$ also showed a center symmetric phase [5]. A perturbation theory analysis on lattice with Wilson fermions and with one small direction agreed with the result $[6,7]$ (see also [8]). The perturbation theory results have also been confirmed by numerical studies on a single site lattice [9].

In this work we describe a perturbative calculation of $\mathrm{QCD}(\mathrm{Adj})$ on a single site lattice with naïve and overlap fermions. The results show that the center symmetry is not restored for naïve fermions but it is restored for overlap fermions for a wide range of Wilson mass values $m$. The study of naïve fermions is motivated since we do not expect doublers on a single site lattice with adjoint fermions. The complete study including lattice simulations is presented in [10].

\section{Eguchi-Kawai model with adjoint fermions}

Eguchi-Kawai model with fermions is defined with the action

$$
S=S^{g}+S^{f}
$$

where

$$
S^{g}=-b N \sum_{\mu \neq v=1}^{4} \operatorname{Tr}\left[U_{\mu} U_{v} U_{\mu}^{\dagger} U_{v}^{\dagger}\right]
$$

and

$$
S^{f}=-f \log \operatorname{det} H_{n, o}=-f \operatorname{Tr} \log H_{n, o},
$$

where $U_{\mu} \in \mathrm{SU}(N)$ are the link matrices, $b=\frac{1}{g^{2} N}$ is the gauge coupling constant, $H_{n, o}$ is the naive or overlap fermion operator, and $f$ is the number of Dirac fermion flavors.

Matrices $V_{\mu}$ are the link matrices in adjoint representation calculated from $U_{\mu}$ as

$$
V^{a b}=\frac{1}{2} \operatorname{Tr}\left[T^{a} U T^{b} U^{\dagger}\right]
$$

where $T^{a}$ are the hermitian generators of $\mathrm{SU}(\mathrm{N})$ normalized as $\operatorname{Tr}\left[T^{a} T^{b}\right]=2 \delta^{a b}$.

Both $H_{n}$ and $H_{o}$ are $4\left(N^{2}-1\right) \times 4\left(N^{2}-1\right)$ hermitian matrices and correspond to naïve Dirac fermions and overlap Dirac fermions respectively. The exact forms are given in [10]. The determinant of $H_{n, o}$ is positive definite and therefore the logarithm is well defined. Furthermore, we can 
define a hermitian matrix $\Sigma$

$$
\begin{aligned}
& \Sigma=\left(\begin{array}{cc}
\sigma_{2} & 0 \\
0 & -\sigma_{2}
\end{array}\right) ; \\
& \Sigma^{\dagger}=\Sigma ; \quad \Sigma^{2}=1,
\end{aligned}
$$

such that,

$$
\Sigma H_{n, o} \Sigma=H_{n, o}^{*}
$$

which implies that all eigenvalues of $H_{n, o}$ are doubly degenerate reflecting the adjoint nature of the fermions. In addition, both naïve and overlap fermions obey chiral symmetry and therefore the eigenvalues of $H_{n, o}$ will come in \pm pairs. Therefore the factor, $f$, in front of $S^{f}$ can be an integer (single Dirac flavor) or half-integer (single Majorana flavor) for all values of $N .^{1}$

\section{Weak coupling expansion}

Our aim is to find out if the $Z_{N}^{4}$ symmetries are broken in the weak coupling limit. We follow [2] and perform the weak coupling analysis by decomposing $U_{\mu}$ according to

$$
U_{\mu}=e^{i a_{\mu}} D_{\mu} e^{-i a_{\mu}} ; \quad D_{\mu}^{i j}=e^{i \theta_{\mu}^{i}} \delta_{i j}
$$

Keeping $\theta_{\mu}^{i}$ fixed, we expand in powers of $a_{\mu}$. The lowest contribution to $S_{g}$ comes from the quadratic term in $a_{\mu}$ [2] and the lowest contribution to $S_{f}$ comes from setting $a_{\mu}=0$.

The computation of the fermion determinant reduces to a free field calculation at this order and the result is

$$
S_{n, o}=-4 f \sum_{i \neq j} \ln \lambda_{n, o}\left(\theta^{i}-\theta^{j}+\phi\right)-4(N-1) f \ln \lambda_{n, o}(\phi),
$$

where $e^{i \phi_{\mu}}$ is the boundary condition in the $\mu$ direction. The eigenvalues, $\pm \lambda(p)$, are two fold degenerate and the explicit expressions are given in [10]. The complete result from fermions and gauge fields is

$$
S=\sum_{i \neq j}\left\{\ln \left[\sum_{\mu} \sin ^{2} \frac{1}{2}\left(\theta_{\mu}^{i}-\theta_{\mu}^{j}\right)\right]-4 f \ln \lambda_{n, o}\left(\theta^{i}-\theta^{j}+\phi\right)\right\}-4(N-1) f \ln \lambda_{n, o}(\phi) .
$$

Independent of the actual values of $\theta_{\mu}^{i}$, the fermion eigenvalues will have $(N-1)$ zero modes with periodic boundary conditions when the mass is set to zero. If all the $\theta_{\mu}^{i}$ are different for each $\mu$, then the fermions should not have exact zero modes when we set $p_{\mu}$ equal to $\left(\theta_{\mu}^{i}-\theta_{\mu}^{j}\right)$ with $i \neq j$. If the fermion spectrum has more than $(N-1)$ zero modes, we will refer to the extra ones as doubler zero modes.

In order to find the minimum of $S$, we consider the Hamiltonian

$$
H=\frac{1}{2} \sum_{\mu, i}\left(\pi_{\mu}^{i}\right)^{2}+\beta S
$$

\footnotetext{
${ }^{1}$ Note that one should have written $\gamma_{5} H_{n, o}$ in (2.3) but this is the same as writing $H_{n, o}$ as long as $f$ is an integer multiple of $\frac{1}{2}$.
} 


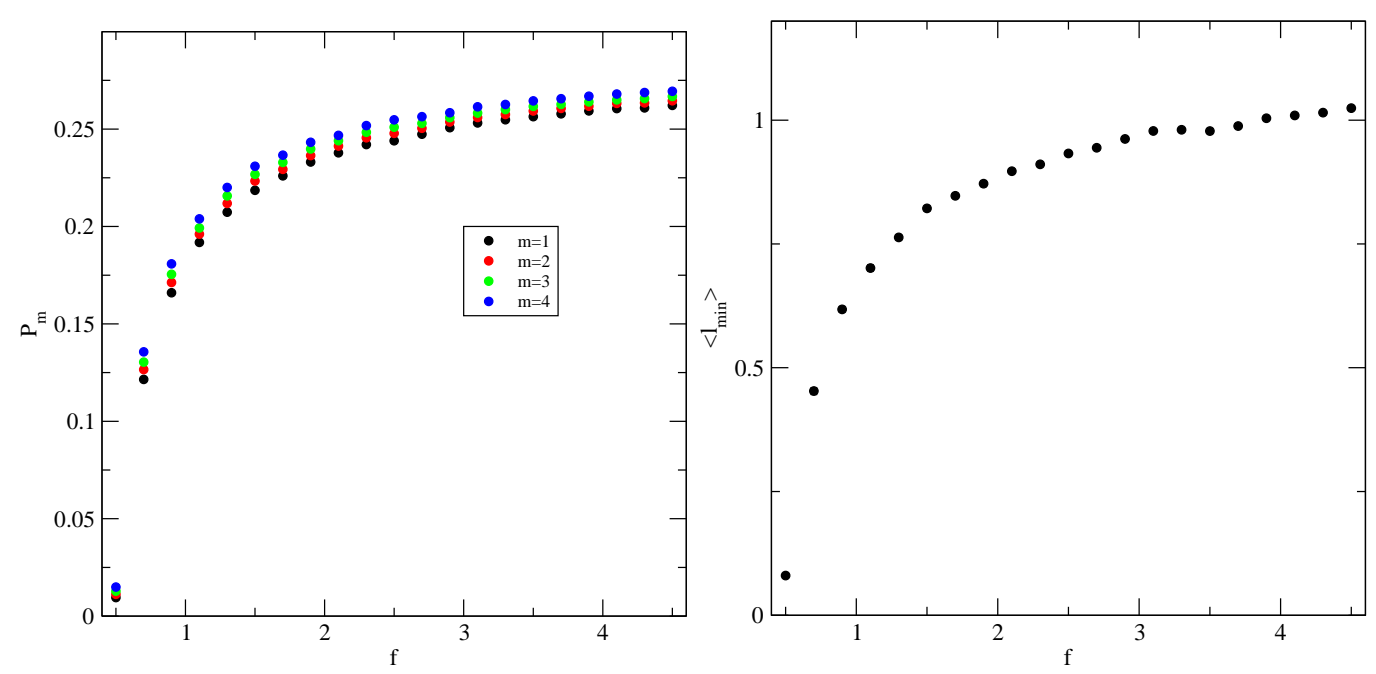

Figure 1: Naïve fermions: Left: $P_{\mu}$ as a function of $f$ at $N=23, \beta=4$, and $\mu=0.01$. Right: Smallest eigenvalue, $\lambda(p)$ with $p_{\mu}=\left(\theta_{\mu}^{i}-\theta_{\mu}^{j}\right)$ and $i \neq j$, for $N=23, \beta=4$, and $\mu=0.01$.

For large $\beta$, the Boltzmann measure $e^{-H}$ will be dominated by the minimum of $S$. We can perform a HMC update of the $\pi, \theta$ system to find this minimum.

A choice for the order parameters associated with the $Z_{N}^{4}$ symmetries is [2]

$$
P_{\mu}=\frac{1}{2}\left(1-\frac{1}{N^{2}}\left|\operatorname{Tr} U_{\mu}\right|^{2}\right)=\frac{1}{N^{2}} \sum_{i, j} \sin ^{2} \frac{1}{2}\left(\theta_{\mu}^{i}-\theta_{\mu}^{j}\right)
$$

The value $P_{\mu}=\frac{1}{2}$ corresponds to unbroken symmetry in $\mu$ direction and $P_{\mu}=0$ totally broken symmetry.

\subsection{Naïve fermions break the $Z_{N}^{4}$ symmetries}

We assume periodic boundary conditions. We pick one value of $N$ and $\beta$ and calculate $P_{\mu}$ as a function of $f$. In order to clearly see symmetry breaking we use rotational symmetry on the lattice and choose to label our directions such that $P_{1}<P_{2}<P_{3}<P_{4}$ for each configuration in our thermalized ensemble. We set $\mu=0.01$ to avoid potential singularities that could occur for the massless case. The plot for $\beta=4$ confirms the breaking of the center symmetry left panel of Fig. 1 . The value is well below $\frac{1}{2}$.

With $N=23$, we expect 22 exact zero modes for $\lambda(p)$ as explained in section 3 . We plot the average of the smallest eigenvalue, $\lambda(p)$ with $p_{\mu}=\left(\theta_{\mu}^{i}-\theta_{\mu}^{j}\right)$ and $i \neq j$, in right panel of Fig. 1. It is clear that this eigenvalue is non-zero for all values of $f$ indicating that there are no doubler zero modes. The reason for the breaking of the $Z_{N}^{4}$ symmetries can be understood by looking at the total action obtained from (3.3):

$$
S=\sum_{i \neq j} \ln \left[\sum_{\mu} \sin ^{2} \frac{1}{2}\left(\theta_{\mu}^{i}-\theta_{\mu}^{j}\right)\right]-2 f \sum_{i \neq j} \ln \left[\mu^{2}+\sum_{\mu} \sin ^{2}\left(\theta_{\mu}^{i}-\theta_{\mu}^{j}\right)\right] .
$$

The fermionic contribution cannot separate $\theta_{\mu}^{i}=\theta_{\mu}^{j}$ from $\theta_{\mu}^{i}=\theta_{\mu}^{j}+\pi$ implying that the fermion contribution alone will result in a distribution of eigenvalues restricted to a width of $\pi$. 

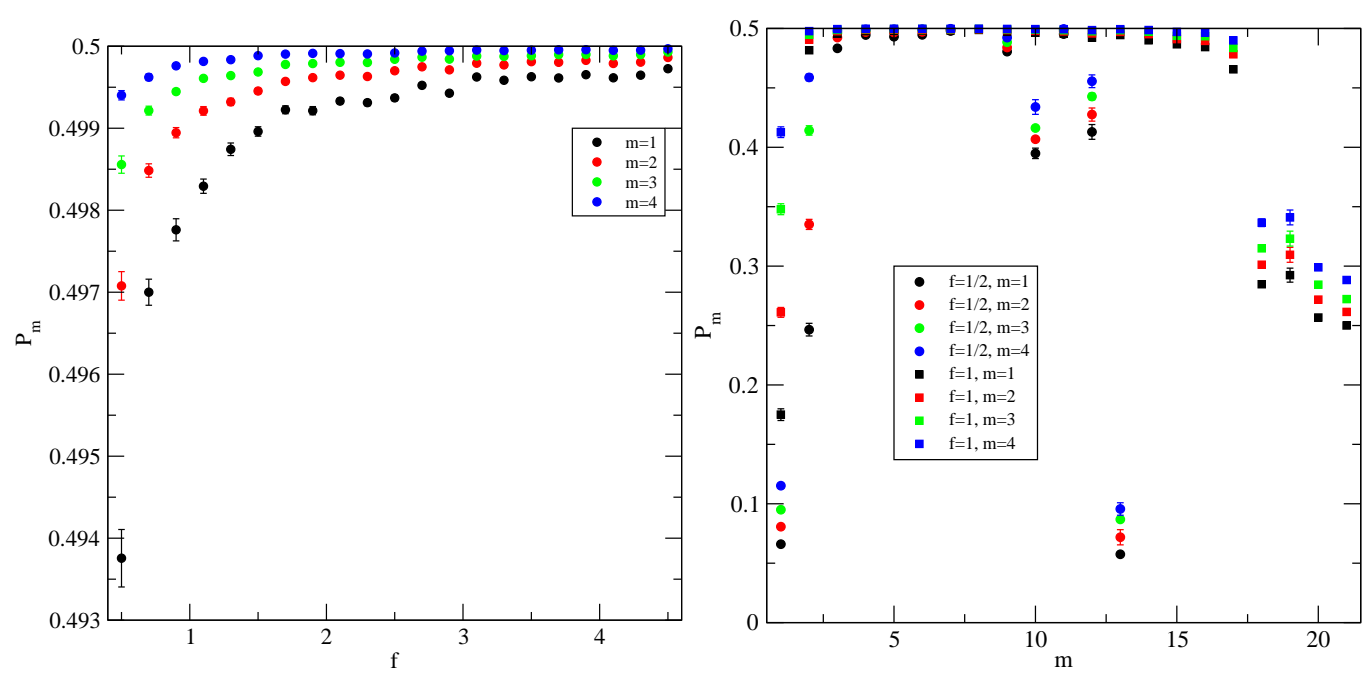

Figure 2: Overlap fermions: Left: Plot of $P_{\mu}$ as a function of $f$ at $N=23, \beta=1$, and $\mu=0.01$. Right:Plot of $P_{\mu}$ as a function of $m$ at $N=23, \beta=1$, and $\mu=0.01$ for two different values of $f$.

\subsection{Overlap fermions do not break the $Z_{N}^{4}$ symmetries}

In contrast to naïve fermions the overlap fermions do not break the $Z_{N}^{4}$ symmetries. A plot of $P_{\mu}$ for several values of $f$ at $N=23$ and $\beta=1$ with $\mu=0.01$ in right panel of Fig. 2 shows this to be the case. The very small deviation close to $f=\frac{1}{2}$ is a consequence of finite $N$ effects. Since we probably are not able to go beyond $N=23$ in the full simulation of the model, this plot will serve as a guide to what one can expect in a full simulation.

Because there are no doubler zero modes, we do not have any restriction on the values for the Wilson mass, $m$, used in the Wilson-Dirac kernel. However, we cannot make it arbitrarily large since one can see by a direct computation that the large $m$ limit of overlap fermions is naïve fermions [11]. A plot of $P_{\mu}$ as a function of $m$ is shown form $f=\frac{1}{2}$ and $f=1$ in right panel of Fig. 2. It indicates that $3 \leq m \leq 8$ is an appropriate range of values of $m$ where the $Z_{N}^{4}$ symmetries are not broken for $f=\frac{1}{2}$ and that range only gets bigger as $f$ increases. A plot of the lowest positive eigenvalue of $H_{w}$ in left panel of Fig. 3 and the smallest eigenvalue, $\lambda_{o}(p)$ with $p_{\mu}=\left(\theta_{\mu}^{i}-\theta_{\mu}^{j}\right)$, $i \neq j$, in right panel of Fig. 3 shows that there are no doubler zero modes in this range of $m$. This range of $m$ can be used for full numerical simulation with overlap fermions.

\subsection{Effect of fermion boundary conditions}

We have assumed periodic boundary conditions for fermions in the previous two subsections. Other choices of boundary conditions that do not generate a $\mathrm{U}(1)$ anomaly amount to replacing $V_{\mu}$ by $V_{\mu} e^{i \frac{2 \pi k_{\mu}}{N}}$ with integer valued $k_{\mu}$ [12]. Physical results are expected to depend on the choice of boundary conditions. This is in contrast to the case of large $N$ gauge theories coupled to fundamental fermions. In that case, the $e^{i \frac{2 \pi k \mu}{N}}$ factor can be absorbed by a change of gauge fields that only changes the Polyakov loop and not the action. If the $Z_{N}$ symmetries are not broken as is the case in the confined phase, this change will not affect physical results. 


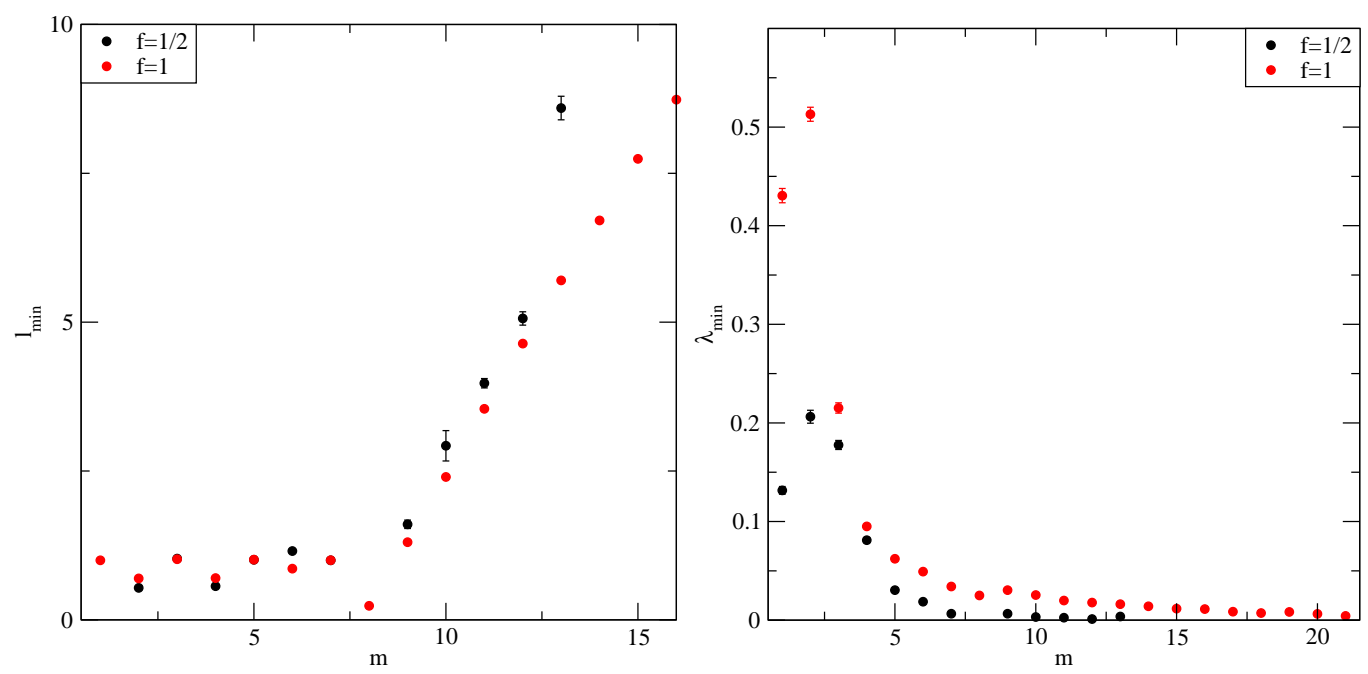

Figure 3: Overlap fermions: Left Plot of the lowest positive eigenvalue of $H$ as a function of $m$ at $N=23$, $\beta=1$, and $\mu=0.01$ for two different values of $f$. Right:Plot of smallest eigenvalue, $\lambda(p)$ with $p_{\mu}=$ $\left(\theta_{\mu}^{i}-\theta_{\mu}^{j}\right)$ and $i \neq j$, for $N=23, \beta=1$, and $\mu=0.01$.

In order to study the effect of boundary conditions on $Z_{N}^{4}$ symmetry breaking, we focus on $f=\frac{1}{2}$ and $f=1$. We set $\phi_{\mu}=0$ for $\mu=2,3,4$ and varied $\phi_{1}$ by setting it to equal to $\frac{2 \pi k}{N}$ with $k$ an integer in the range $0 \leq k<N / 2$. The plot of $P_{\mu}$ as a function of $\phi_{1}$ is shown in Fig. 4. We see that the $Z_{N}$ symmetry in the $\mu=1$ direction is broken if $\phi_{1}>\frac{\pi}{2}$. This seems to be the case in the limit of large $N$ and seems to be roughly independent of $f$. Furthermore, the $Z_{N}$ symmetries in the other three directions with periodic boundary conditions are not broken. This result could help us force feed momentum for quarks in the adjoint representation. In order to pursue this, we need to study the effect of $\phi_{1}$ on the chiral condensate and see if chiral symmetry is restored when the $Z_{N}$ symmetry is broken and if the chiral condensate is independent of the value of $\phi_{1}$ when the $Z_{N}$ symmetry is not broken.

\section{Discussion}

We have provided perturbation theory arguments that the $Z_{N}^{4}$ symmetry is restored in the large $N$ limit for overlap fermions but not for naïve fermions. The details of perturbation theory calculations with framework to HMC-lattice simulations are given in [10]. The natural continuation is to perform lattice calculations on several $N$ and calculate physical observables, e.g., string tension and chiral condensate.

\section{Acknowledgments}

The authors acknowledge partial support by the NSF under grant number PHY-0854744. AH also acknowledges partial support by the U.S. DOE grant under Contract DE-FG02-01ER41172. 


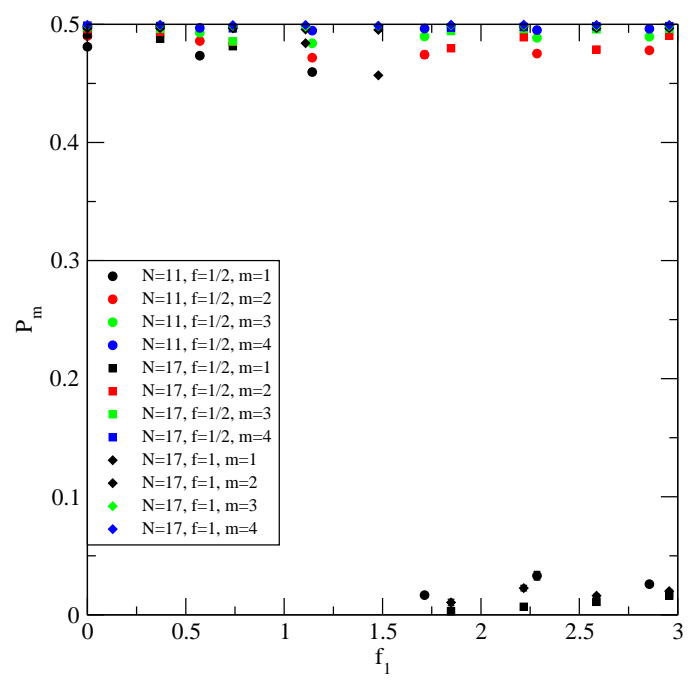

Figure 4: Overlap fermions: Plot of $P_{\mu}$ as a function of $\phi_{1}$ for three different values of $(f, N)$ with $\beta=1$ and $\mu=0.01$.

\section{References}

[1] T. Eguchi and H. Kawai, Phys. Rev. Lett. 48, 1063 (1982).

[2] G. Bhanot, U. M. Heller and H. Neuberger, Phys. Lett. B 113, 47 (1982).

[3] R.L. Mkrtchyan and S.B. Khokhlachëv, Pis'ma Zh. Eksp. Teor. Fiz. 37 No 3, 160-161 (1983).

[4] P. Kovtun, M. Unsal and L. G. Yaffe, JHEP 0706, 019 (2007) [arXiv:hep-th/0702021].

[5] T. J. Hollowood and J. C. Myers, arXiv:0907.3665 [hep-th].

[6] B. Bringoltz, JHEP 0906, 091 (2009) [arXiv:0905.2406 [hep-lat]].

[7] B. Bringoltz, arXiv:0911.0352 [hep-lat].

[8] P. F. Bedaque, M. I. Buchoff, A. Cherman and R. P. Springer, arXiv:0904.0277 [hep-th].

[9] B. Bringoltz and S. R. Sharpe, Phys. Rev. D 80, 065031 (2009) [arXiv:0906.3538 [hep-lat]].

[10] A. Hietanen and R. Narayanan, arXiv:0911.2449 [hep-lat]

[11] R. Narayanan and H. Neuberger, Nucl. Phys. B 443, 305 (1995) [arXiv:hep-th/9411108].

[12] E. Poppitz and M. Unsal, JHEP 0903, 027 (2009) [arXiv:0812.2085 [hep-th]]. 